ISSN: 2644-1381

\title{
P Value and Statistical Significance
}

\author{
Vidya Laxmi K* \\ Department of Statistics, Manasagangotri, University of Mysore, Mysore, India
}

Received: September 14, 2018; Published:

*Corresponding author: Vidya laxmi K, Department of Statistics, Manasagangotri, University of Mysore, Mysore, India

\begin{abstract}
The practice of reporting P-values is commonplace in applied research. Presenting the result of a test only as the rejection or acceptance of the null hypothesis at a certain level of significance, does not make full use of the information available from the observed value of the test statistic. Rather P-values have been used in the place of hypothesis tests as a means of giving more information about the relationship between the data and the hypothesis. In this brief note we discuss how to obtain P-values with R codes.
\end{abstract}

Keywords: Hypothesis; Distribution; P-value

\section{Introduction}

Very often in practice we are called upon to make decisions about populations on the basis of sample data. In attempting to reach decisions, it is useful to make assumptions or guesses about the populations involved. Such assumptions which may or may not be true, are called statistical hypotheses and in general are statements about the population parameters. The entire procedure of testing of hypothesis that consists of setting up what is called a 'Null hypothesis' and testing it. R.A. Fisher quotes, 'Every experiment may be said to exist only in order to give the facts about a chance of disproving the null hypothesis'. So, what is this null hypothesis?". For example, if we consider the measurements on weights of newborn babies, then the observations on these measurements follows Normal distribution is a null hypothesis [1]. Suppose the measurements denoted by a random variable $\mathrm{X}$ that is thought to have a normal distribution with mean $\mu$ and variance 1 , denoted by $N(\mu, 1)$. The usual types of hypotheses concerning mean $\mu$ in which one is interested include $H_{0}: \mu=\mu_{0}$ versus $H_{1}: \mu_{6}=$ $\mu_{0}$ (two-tailed hypothesis) and $H_{0}: \mu \leq \mu_{0}$ versus $H_{1}: \mu>\mu_{0}$ and $H_{0}: \mu$ $\geq \mu 0$ versus $H_{1}: \mu<\mu_{0}$ (one-tailed hypothesis). So null hypothesis $\mathrm{H}_{0}$ is a hypothesis which is tested for possible rejection under the assumption that it is true.

In general, a procedure for the problem of testing of significance of a hypothesis is as follows: Given the sample point $\mathrm{x}=\left(\mathrm{x}_{1}, \mathrm{x}_{2}, \ldots\right.$. , $\mathrm{x}_{\mathrm{n}}$ ), find a decision ru. le that will lead to a decision [2].

\section{$P$ Value and Statistical Significance}

To reject or fail to reject the null hypothesis $H_{0}: \theta \in \Theta_{0}$ in favor of the alternative hypothesis $H_{1}: \theta \in \Theta_{1}=\Theta-\Theta_{0}$. This decision rule is based on a test statistic whose probability distribution when $\mathrm{HO}$ is true is known, at least approximately. Calculate the value of the test statistic for the available data [3]. If the test statistic is in the extreme of its probability distribution under the null hypothesis, there is evidence that the null hypothesis is rejected. More quantitatively, we calculate from the distribution of the test statistic, the probability $\mathrm{P}$ that a deviation would arise by chance as or more extreme than that actually observed, the $\mathrm{H}_{0}$ being true. This value of $\mathrm{P}$ is called the significance level achieved from the sample data or P-value. There are several ways to define P-values [4]. It is the probability of observing under $\mathrm{H}_{0}$ a sample outcome at least as extreme as the one observed. One could define P-value as the greatest lower bound on the set of all significance levels $\alpha$ such that we would reject $\mathrm{H}_{0}$ at level $\alpha$. P-value is a value satisfying $0 \leq \mathrm{P} \leq 1$ for every sample point $\mathrm{X}$. A P-value is valid if $\mathrm{P}_{\theta}(\mathrm{p} \leq \alpha) \leq \alpha$. For fixed sample data $\mathrm{X}=\mathrm{x}$ it changes for different hypotheses. Let $\mathrm{T}(\mathrm{X})$ be a test statistic such that large values of $T$ give evidence that $H_{1}$ is true. For each sample point $\mathrm{x}, \mathrm{P}$-value is defined as $\mathrm{p}$-value $=\sup \mathrm{P}_{\theta}(\mathrm{T}(\mathrm{X})$ $\geq \mathrm{T}(\mathrm{x})$ ). $\theta \in \Theta_{0}$. Fisher writes: "The value for which P-value $=0.05$, or 1 in 20 , is 1.96 or nearly 2 ; it is convenient to take this point as a limit in judging whether a deviation ought to be considered significant or not. Deviations exceeding twice the standard deviation are thus 
formally regarded as significant". Thus, for a given $\alpha$, we reject $\mathrm{H} 0$ if $\mathrm{P}$-value $\leq \alpha$ and do not reject $\mathrm{H}_{0}$ if $\mathrm{P}$-value $>\alpha$. In the two-tailed case, if the distribution of the test statistic is symmetric, one-tailed P-value is doubled to obtain P-value. If the distribution of the test statistic is not symmetric, the P-value is not well defined in twotailed case, although many authors recommend doubling the onesided P-value.

Examples: Let $\mathrm{X}_{1}, \ldots, \mathrm{X}_{\mathrm{n}}$ be a random sample from a $\mathrm{N}\left(\mu, \sigma^{2}\right)$ population. If we want to test $H_{0}: \mu \leq \mu_{0}$ versus $H_{1}: \mu>\mu_{0}$ when $\sigma$ unknown, test procedure is to reject $H_{0}$ for large values of $T(x)=\frac{\bar{x}-\mu_{0}}{s / \sqrt{(n)}}$ which has Student's t distribution with $\mathrm{n}-1$ degrees of freedom when $\mathrm{H}_{0}$ is true. Thus, the $\mathrm{P}$ value for this one-sided test is $\mathrm{P}$-value $=\mathrm{P}(\mathrm{Tn}-1 \geq \mathrm{T}(\mathrm{x}))$. Again, if we want to test $\mathrm{H}_{0}: \mu=\mu_{0}$ versus $\mathrm{H}_{1}: \mu_{6}=\mu_{0}$ then $T(x)=\frac{\left|\bar{x}-\mu_{0}\right|}{s / \sqrt{(n)}}$ and P-value $=2 \mathrm{P}(\mathrm{Tn}-1 \geq \mathrm{T}(\mathrm{x})) . \mathrm{R}$ codes for obtaining these P-values are "1-pt(T(x), df)" and "2*(1- pt(T(x), df))" respectively where $\mathrm{df}$ is the degrees of freedom. If we want to test these hypotheses when $\sigma$ known, test procedure is to reject $\mathrm{H}_{0}$ for large values of $z=\frac{\bar{x}-\mu_{0}}{\sigma / \sqrt{(n)}}$ which has standard normal distribution when $\mathrm{H}_{0}$ is true. Then $\mathrm{P}$-value= $\mathrm{P}(\mathrm{Z} \alpha \geq \mathrm{Z})$ where $\mathrm{Z} \alpha$ is the critical value of $\mathrm{Z}$ for a given level of significance $\alpha$. R codes for obtaining these P-values are "1-pnorm(Z)" and "2*(1- p norm(Z)) or $2 *$ norm(abs(Z))" respectively. Similarly, for testing homogeneity of variances Chi-square $\left(\chi^{2}\right)$ test statistic.

\section{P Value and Statistical Significance}

And $\mathrm{F}$ test statistics will be used. R codes of P-values for these tests are "1-pchisq $\left(\chi^{2}, \mathrm{df}\right)$ " and "1-pf ( $\left.\mathrm{F}, \mathrm{df} 1, \mathrm{df} 2\right)$ " respectively where $\mathrm{df}$ is degrees of freedom, df1 is the degrees of freedom for numerator and df2 is the degrees of freedom for the denominator.

\section{Conclusion}

Nowadays reporting of $p$-values is very common in applied fields. The most important conclusion is that, for testing the hypotheses, P-values should not be used directly, because they are easily misinterpreted. From the Bayesian perspective, P-values overstate the evidence against the null hypothesis and other methods to adduce evidence (likelihood ratios) may be of more utility. Finally, in many scenarios P-values can distract or even mislead, either a nonsignificant result wrongly interpreted as a confidence statement in support of the null hypothesis or a significant P-value that is taken as proof of an effect. Thus, there would appear to be considerable virtue in reporting both $\mathrm{P}$-values and confidence interval (CI), on the basis that singular statements such as $\mathrm{P}_{\dot{\imath}} 0.05$, or $\mathrm{P}=$ Non-Significant, convey little useful information, although for a $100(1-\alpha) \% \mathrm{CI}$, it must be remembered that any violation of the assumptions that effect the true value of effect CI precision. From the Bayesian perspective, Lindley has summarized the position thus: significance tests, as inference procedures, are better replaced by estimation methods it is better to quote a confidence interval. Finally, p-values should be retained for a limited role as part of the statistical significance approaches.

\section{References}

1. Berkson J (1942) Tests of significance considered as evidence. Journal of American Statistical Association 37(219): 325-335.

2. Cox DR (1982) Statistical significance tests. Br J clin Pharmac 14(3): 325-331.

3. Rohatgi VK, AK Saleh (2011) An introduction to probability and statistics. ( $2^{\text {nd }}$ edn) Wiley publications, USA.

4. Schervish MJ (1996) P Values: What They Are and What They Are Not. The Americanz Statistician 50(3): 203-206.
This work is licensed under Creative Commons Attribution 4.0 License

To Submit Your Article Click Here: $\quad$ Submit Article

DOI: 10.32474/CTBB.2018.01.000102

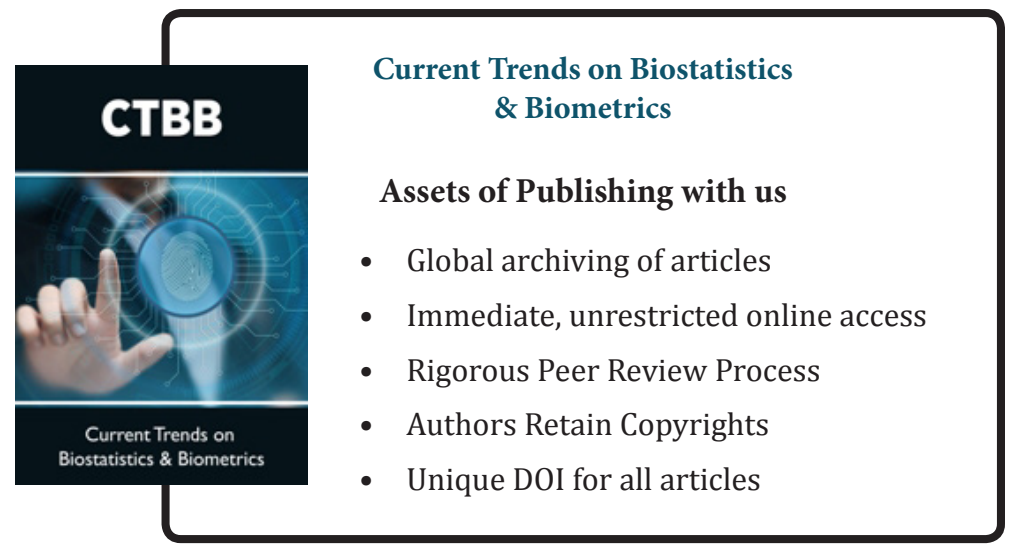

